\title{
Risk of Psychiatric Disorders Following Primary Sjögren Syndrome: A Nationwide Population-based Retrospective Cohort Study
}

\author{
Cheng-Che Shen, Albert C. Yang, Benjamin Ing-Tiau Kuo, and Shih-Jen Tsai
}

\begin{abstract}
Objective. Primary Sjögren syndrome (pSS) is a chronic autoimmune disease. A clear temporal causal relationship between pSS and psychiatric disorders has not been well established. We used a nationwide population-based retrospective cohort study to explore the relationship between pSS and the subsequent development of psychiatric disorders.

Methods. We identified subjects who were newly diagnosed with pSS between January 1, 2000, and December 31, 2008, in the Taiwan National Health Insurance (NHI) Research Database. A comparison cohort was constructed for patients without pSS. There were 2686 patients with pSS and 10,744 matched controls observed until diagnosed with psychiatric disorders or until death, withdrawal from the NHI system, or December 31, 2009. The Institutional Review Board of Taipei Veterans General Hospital approved this study (2012-12-013BC).

Results. The adjusted HR of depressive disorder, anxiety disorder, and sleep disorder in subjects with pSS were significantly higher at $1.829,1.856$, and 1.967 than those of the controls during the followup. We found that pSS might increase the risk of subsequent newly diagnosed depressive disorder, anxiety disorder, and sleep disorder that may impair life quality.

Conclusion. Our findings highlight the need for psychiatric evaluation and intervention for patients with pSS. (First Release May 15 2015; J Rheumatol 2015;42:1203-8; doi:10.3899/jrheum.141361)
\end{abstract}

Key Indexing Terms:

PRIMARY SJÖGREN SYNDROME ANXIETY DISORDER

\section{DEPRESSIVE DISORDER} SLEEP DISORDER
Primary Sjögren syndrome (pSS) is a systemic autoimmune disease characterized by chronic inflammation and lymphocytic infiltration of exocrine glands that results in dry eyes (xerophthalmia) and dry mouth (xerostomia $)^{1,2}$. Its estimated

From the Department of Psychiatry, Chiayi Branch, Taichung Veterans General Hospital; Department of Information Management, National Chung-Cheng University, Chiayi; Department of Psychiatry, and Department of Research and Medication, Taipei Veterans General Hospital; School of Medicine, National Yang-Ming University, Taipei, Taiwan.

Supported by grant NSC 101-2314-B-075-040 from the National Science Council, Taiwan, and grant V103C-048 from the Taipei Veterans General Hospital. The study was based on data from the National Health Insurance (NHI) Research Database (provided by the Bureau of NHI of the

Department of Health, Taiwan, and managed by the National Health

Research Institute, Taiwan). The interpretation and conclusions contained in this article do not represent those of the Bureau of NHI, the Department of Health, or the National Health Research Institute.

C.C. Shen, MD, Department of Psychiatry, Chiayi Branch, Taichung Veterans General Hospital; A.C. Yang, MD, PhD, Department of Psychiatry, Taipei Veterans General Hospital, and School of Medicine, National Yang-Ming University; B.I. Kuo, MD, PhD, Department of Research and Medication, Taipei Veterans General Hospital, and School of Medicine, National Yang-Ming University; S.J. Tsai, MD, Department of Psychiatry, Taipei Veterans General Hospital, and School of Medicine, National Yang-Ming University.

Address correspondence to Dr. S.J. Tsai, Department of Psychiatry, Taipei Veterans General Hospital, No. 201, Shih-Pai Road, Sec. 2, 11217, Taipei,

Taiwan.E-mail:tsai610913@gmail.com

Accepted for publication March 16, 2015. prevalence varies between $0.5 \%$ and $1 \%$ of the general population ${ }^{2}$. The disease is associated with a high prevalence in women, and although it can develop at any age, it is most prevalent after age $40^{1,2}$. In addition to the dry eyes and dry mouth that characterize pSS, arthralgia, myalgia, interstitial nephritis, and interstitial pneumonitis are also commonly found in subjects with $\mathrm{pSS}^{1,2}$.

Chronic diseases are usually accompanied by psychological abnormalities ${ }^{3}$. Further, symptoms of dry burning mouth have been associated with depression and anxiety ${ }^{4}$. However, there is a limited number of published studies on the epidemiology of psychiatric comorbidity in patients with SS. In 1994, Utset, et al investigated depressive symptoms in 349 patients with systemic lupus erythematosus (SLE) ${ }^{5}$. They found patients with secondary SS who had an adjusted OR of 2.97 for depressive symptoms and suggested that depression in patients with SLE does not occur simply as a response to social stresses and may be a manifestation of autoimmune disease in some patients ${ }^{5}$. In a cross-sectional study of 80 adult UK subjects (40 diagnosed with pSS and 40 controls with no history of chronic illness), patients with pSS appeared to be at increased risk from clinical depression, but not anxiety ${ }^{6}$. Regarding a study with a questionnaire [the Hospital Anxiety and Depression Scale (HADS)], it was found that the patients with pSS had a significantly higher

Personal non-commercial use only. The Journal of Rheumatology Copyright (c) 2015. All rights reserved. 
scoring rate for "possible" clinical anxiety (48\%) and for "possible" clinical depression (32\%) compared with reference groups ${ }^{7}$. In a nationwide population-based study of comorbidity profiles among patients with pSS in Taiwan, it was found that patients with pSS had a higher OR of mental illness, including depression (OR 2.57) and psychoses (OR $2.15)$, than the comparison group ${ }^{8}$. Comorbid depression in subjects with pSS has been reported to lead to functional impairment, decreased quality of 1 ife $\mathrm{e}^{9,10,11}$, fatigue ${ }^{12}$, and work disability ${ }^{13,14}$.

Although the above-mentioned research has provided insight into the association between pSS and comorbid psychiatric disorders, most of these study results are based on psychiatric ratings rather than diagnosis by a psychiatrist, or based on cross-sectional study design and lack a longitudinal perspective. Further, the small sample size of most of the above studies prevents generalization of the findings. In response to the lack of longitudinal studies concerning the association between pSS and the subsequent risk of psychiatric disorders, and based on the hypothesis that pSS might convey a higher risk for developing subsequent psychiatric disorders, we designed a nationwide population-based retrospective cohort study to investigate the possible link between these 2 illnesses.

\section{MATERIALS AND METHODS}

Data sources. Instituted in 1995, the National Health Insurance (NHI) program is a mandatory health insurance program that offers comprehensive medical care coverage, including outpatient, inpatient, emergency, and traditional Chinese medicine to all residents of Taiwan, with a coverage rate of up to $98 \%{ }^{15}$. The NHI Research Database (NHIRD) contains comprehensive information regarding clinical visits, including prescription details and diagnostic codes based on the A code and International Classification of Diseases, 9th ed, Clinical Modification (ICD-9-CM). The NHIRD is managed by the National Health Research Institutes (NHRI), and confidentiality is maintained according to the directives of the Bureau of NHI. The data source for our study was the Longitudinal Health Insurance Database 2005 (LHID 2005), which is a dataset released by the NHRI that contains all original claims data for 1 million randomly selected beneficiaries in the 2005 Registry of Beneficiaries.

Ethics statement. The Institutional Review Board of Taipei Veterans General Hospital approved our study (2012-12-013BC). Written consent from the study patients was not obtained because the NHI dataset consists of de-identified secondary data for research purposes and the Institutional Review Board of Taipei Veterans General Hospital issued a formal written waiver for the need for consent.

Study population. Using data extracted from the LHID 2005, we conducted a retrospective cohort study of patients who were newly diagnosed with pSS (ICD-9-CM code: 710.2) between January 1, 2000, and December 31, 2008. The diagnoses were considered reliable because the features of dryness of the eyes and mouth and blood antibody testing (such as antinuclear antibodies) were required for initial diagnosis of pSS in Taiwan. To further assure validity of diagnosis and patient homogeneity, we selected only patients who had at least 2 consensus pSS diagnoses for the study group ${ }^{16}$ We excluded patients who were diagnosed with pSS between January 1, 1996, and December 31, 1999. We also excluded patients who were diagnosed with psychiatric disorders (A codes: A210-A219; ICD-9-CM codes: 290-319) before they were diagnosed with pSS. For each patient with pSS included in the final cohort, 4 age- and sex-matched control patients without psychiatric disorder were randomly selected from the LHID 2005 All patients with pSS and control patients were observed until diagnosed with schizophrenia (ICD-9-CM code: 295), depressive disorder (ICD-9-CM codes: $296.2,296.3,300.4$, and 311), bipolar disorder (ICD-9-CM codes: 296.0, 296.1, 296.4, 296.5, 296.6, 296.7, 296.8, 296.80, and 296.89), anxiety disorder (ICD-9-CM codes: 300.0, 300.2, 300.3, 308.3, and 309.81), or sleep disorder (ICD-9-CM codes: 780.5, 307.4; excluding 780.51, 780.53, 780.57) by a psychiatrist, or until death, withdrawal from the NHI system, or December 31,2009. The primary clinical outcomes assessed were psychiatrist-diagnosed schizophrenia, depressive disorder, bipolar disorder, anxiety disorder, and sleep disorder.

Statistical analysis. The incidence of newly diagnosed schizophrenia, depressive disorder, bipolar disorder, anxiety disorder, or sleep disorder in the patients with pSS and control patients was calculated, and independent Student $t$ tests and chi-square tests were conducted to examine the differences in the characteristics between the patients with pSS and control patients. To investigate potential surveillance bias, subgroups were stratified according to the duration since pSS diagnosis. A Cox proportional hazards regression model was constructed to calculate the HR of schizophrenia, depressive disorder, bipolar disorder, anxiety disorder, and sleep disorder of the pSS cohort and control cohort. We also tested the proportionality of hazards in our work by using the Scaled Schoenfeld Residuals test.

SAS statistical software for Windows, Version 9.3 (SAS Institute) was used for data extraction, computation, linkage, processing, and sampling. All other statistical analyses were performed using the SPSS statistical software for Windows, Version 20 (IBM). The results of comparisons with a $p$ value less than 0.05 were considered to indicate a statistically significant relationship.

\section{RESULTS}

Our study sample was composed of 2686 patients with pSS (28.3\% men) and 10,744 control patients without pSS. The comparisons of demographic and clinical variables between the patients with pSS and control patients are presented in Table 1. The median age of the patients with pSS was 45.31 years [interquartile range (IQR) 32.99-57.98] and the median followup duration was 4.70 years (IQR 2.93-7.17).

During the followup period, 215 patients with pSS $(8.00 \%)$ and 475 control patients $(4.42 \%)$ were diagnosed with psychiatric disorders $(\mathrm{p}<0.001)$. The most common subsequent psychiatric disorders in patients with pSS were depressive disorder in 85 patients (3.2\%), anxiety disorder in 67 patients $(2.5 \%)$, and sleep disorder in 55 patients $(2.0 \%)$. Overall, significantly higher incidences of depressive disorder $(\mathrm{p}<0.001)$ and anxiety disorder $(\mathrm{p}<0.001)$ were observed in patients with pSS than in the control patients.

In addition, a Cox proportional hazards regression analysis was conducted to calculate the HR of the newly diagnosed psychiatric disorders for the patients with pSS compared with the matched controls (Table 2). The results indicated that patients with pSS exhibited a markedly higher risk for subsequent depressive disorder (adjusted HR 1.829, 95\% CI 1.400-2.391), anxiety disorder (adjusted HR $1.856,95 \%$ CI 1.384-2.489), and sleep disorder (adjusted HR 1.967, 95\% CI 1.405-2.754) after adjusting for age, sex, comorbidities, urbanization, and monthly income. The results of testing the proportionality of hazards in our work showed that there was no evidence that the proportional hazards assumption was

Personal non-commercial use only. The Journal of Rheumatology Copyright $\subset$ $\subset$ 2015. All rights reserved 
Table 1. Characteristics of patients with pSS and control subjects. Values are n (\%) unless otherwise specified.

\begin{tabular}{|c|c|c|c|}
\hline Characteristics & $\mathrm{pSS}, \mathrm{n}=2686$ & Control, $\mathrm{n}=10,744$ & $\mathrm{p}$ \\
\hline Age, yrs, median (IQR) & $45.31(32.99-57.98)$ & $45.26(32.93-57.99)$ & 0.996 \\
\hline \multicolumn{4}{|l|}{ Age distribution } \\
\hline $20-39$ & $1068(39.8)$ & $4272(39.8)$ & \multirow[t]{3}{*}{0.946} \\
\hline $40-59$ & $1030(38.3)$ & $4120(38.3)$ & \\
\hline$\geq 60$ & $588(21.9)$ & $2352(21.9)$ & \\
\hline \multicolumn{4}{|l|}{ Sex } \\
\hline Male & $760(28.3)$ & $3040(28.3)$ & \multirow[t]{2}{*}{$>0.999$} \\
\hline Female & $1926(71.7)$ & $7704(71.7)$ & \\
\hline \multicolumn{4}{|l|}{ Income } \\
\hline Low & $1098(40.9)$ & $5194(48.3)$ & \multirow[t]{3}{*}{$<0.001 *$} \\
\hline Medium & $1050(39.1)$ & $4175(38.9)$ & \\
\hline High & $538(20.0)$ & $1375(12.8)$ & \\
\hline \multicolumn{4}{|l|}{ Degree of urbanization } \\
\hline Urban & $1695(63.1)$ & $6729(62.6)$ & \multirow[t]{3}{*}{0.170} \\
\hline Suburban & $754(28.1)$ & $3152(29.3)$ & \\
\hline Rural & $199(7.4)$ & $863(8.0)$ & \\
\hline \multicolumn{4}{|l|}{ Comorbidities } \\
\hline Hypertension & $517(19.2)$ & $1916(17.8)$ & 0.092 \\
\hline Diabetes mellitus & $348(13.0)$ & $1138(10.6)$ & $0.001 *$ \\
\hline Dyslipidemia & $499(18.6)$ & $1341(12.5)$ & $<0.001 *$ \\
\hline Coronary artery disease & $22(0.8)$ & $71(0.7)$ & 0.367 \\
\hline Congestive heart failure & $66(2.2)$ & $202(1.9)$ & 0.241 \\
\hline Chronic pulmonary disease & $431(16.0)$ & $1031(9.6)$ & $0.001 *$ \\
\hline Malignancy & $58(2.2)$ & $193(1.8)$ & 0.231 \\
\hline Followup, yrs, median (IQR) & $4.70(2.93-7.17)$ & $4.70(2.93-0.17)$ & 0.866 \\
\hline Newly diagnosed psychiatric disorders & $215(8.00)$ & $475(4.4)$ & $<0.001 *$ \\
\hline Schizophrenia & $2(0.1)$ & $24(0.2)$ & 0.143 \\
\hline Bipolar disorder & $6(0.2)$ & $15(0.1)$ & 0.409 \\
\hline Depressive disorder & $85(3.2)$ & $174(1.6)$ & $<0.001 *$ \\
\hline Anxiety disorder & $67(2.5)$ & $145(1.3)$ & $<0.001 *$ \\
\hline Sleep disorder & $55(2.0)$ & $117(1.1)$ & $<0.001 *$ \\
\hline
\end{tabular}

* Statistical significance. pSS: primary Sjögren syndrome; IQR: interquartile range.

Table 2. HR of time until psychiatric disorders between patients with pSS and control subjects during a 10-year followup period.

\begin{tabular}{lcc}
\hline Psychiatric Disorders & Crude HR (95\% CI) & Adjusted HR (95\% CI)* \\
\hline Schizophrenia & $0.334(0.079-1.412)$ & $0.370(0.087-1.579)$ \\
Bipolar disorder & $1.603(0.622-4.133)$ & $1.886(0.726-4.902)$ \\
Depressive disorder & $1.982(1.529-2.569)^{* *}$ & $1.829(1.400-2.391)^{* *}$ \\
Anxiety disorder & $1.861(1.393-2.486)^{* *}$ & $1.856(1.384-2.489)^{* *}$ \\
Sleep disorder & $1.980(1.430-2.742)^{* *}$ & $1.967(1.405-2.754)^{* *}$ \\
\hline
\end{tabular}

* Adjusted for age, sex, hypertension, diabetes mellitus, dyslipidemia, coronary artery disease, congestive heart failure, chronic pulmonary disease, malignancy, urbanization, and income. ** Statistical significance. pSS: primary Sjögren syndrome.

violated in any Cox regression model in our study (supplementary table available from the authors on request).

Further, a subanalysis based on the duration of followup revealed that the risk of newly diagnosed depressive disorder and sleep disorder became significantly elevated only between the first and the fifth year following a pSS diagnosis. On the other hand, the risk of newly diagnosed anxiety disorder increased not only within the first year, but also between the first and the fifth year following a pSS diagnosis. The results of the subanalysis are summarized in Table 3.

Finally, we performed a Cox proportional hazards regression model to identify variables that predicted psychiatric disorders in patients with pSS. The results showed that age, sex, and comorbidities were not significant prognostic factors for psychiatric disorders in patients with pSS (data not shown).

\section{DISCUSSION}

In the present study, patients with pSS had an increased risk of developing depression, anxiety, and sleep disorders compared with age- and sex-matched controls. Further, our analysis showed that diabetes mellitus, dyslipidemia, and chronic pulmonary disease were more prevalent in patients with pSS than in patients without pSS (Table 1), which is consistent with the results of previous studies ${ }^{8,17,18}$, strengthening the reliability of our findings.

Several studies have confirmed that depression is a common comorbidity in patients with $\mathrm{pSS}^{5,6,7,8}$. However, most of the studies used rating scales for depression evaluation. In addition, little empirical evidence has been found to

$$
\text { Personal non-commercial use only. The Journal of Rheumatology Copyright } \subset \text { (2015. All rights reserved. }
$$


Table 3. Number of newly diagnosed depressive and anxiety disorders between patients with pSS and control subjects, stratified by followup duration.

\begin{tabular}{lccccc}
\hline \multirow{2}{*}{$\begin{array}{l}\text { Followup } \\
\text { Duration, Yr }\end{array}$} & $\begin{array}{c}\text { Patients with pSS } \\
\text { No. Depressive } \\
\text { Disorders }\end{array}$ & $\begin{array}{c}\text { Per } 1000 \\
\text { Person-Yrs }\end{array}$ & $\begin{array}{c}\text { Po. Depressive } \\
\text { Disorders }\end{array}$ & $\begin{array}{c}\text { Per } 1000 \\
\text { Person-Yrs }\end{array}$ & \\
\hline Overall & 85 & 6.39 & 174 & 3.23 & $1.98(1.51-2.58)^{*}$ \\
$0-1$ & 12 & 4.48 & 22 & 2.05 & $2.19(0.99-4.61)$ \\
$1-5$ & 55 & 5.29 & 102 & 2.42 & $2.18(1.54-3.05)^{*}$ \\
$\geq 5$ & 18 & 1.35 & 50 & 0.93 & $1.46(0.80-2.54)$ \\
& No. Anxiety & Per 1000 & No. Anxiety & Per 1000 & \\
Overall & Disorders & Person-Yrs & Disorders & Person-Yrs & \\
$0-1$ & 67 & 5.00 & 145 & 2.69 & $1.86(1.37-2.50)^{*}$ \\
$1-5$ & 10 & 3.73 & 16 & 1.49 & $2.50(1.02-5.88)^{*}$ \\
$\geq 5$ & 44 & 4.21 & 92 & 2.19 & $1.93(1.31-2.79)^{*}$ \\
& 13 & 0.97 & 37 & 0.69 & $1.41(0.69-2.72)$ \\
Overall & No. Sleep & Per 1000 & No. Sleep & Per 1000 & \\
$0-1$ & Disorders & Person-Yrs & Disorders & Person-Yrs & \\
$1-5$ & 55 & 4.10 & 117 & 2.16 & $1.89(1.35-2.63)^{*}$ \\
$\geq 5$ & 8 & 2.99 & 16 & 1.49 & $2.00(0.74-4.96)$ \\
& 34 & 3.25 & 67 & 1.59 & $2.04(1.31-3.13)^{*}$ \\
\hline
\end{tabular}

* Statistical significance. pSS: primary Sjögren syndrome; RR: risk ratio.

support a clearer association between the pSS and subsequent depressive disorders. Our nationwide population-based retrospective cohort study using diagnosis by psychiatrists indicated that, after adjusting for covariates, pSS subjects have 1.829 times greater risk of developing depressive disorder than the comparison cohort (Table 2). We speculate that there are at least 4 plausible mechanisms for the increased risk of subsequent depressive disorder in patients with pSS. First, a study has demonstrated that patients with pSS presented higher rates of general psychological distress compared with SLE and healthy participants ${ }^{19}$. In addition, a higher number of patients with pSS reported distinct personality traits (neuroticism, psychoticism, and obsessiveness) that make it harder to manage stress ${ }^{20}$. Both high levels of psychological distress and distinct pattern of personality traits may have potential involvement in depression development in pSS subjects. Second, in some cases it is likely that depression is mediated by the physical discomfort of pSS. In a study of 90 patients with pSS, there was a significant correlation between the intensity of oral dryness and the quality of anxiety and depression measured by the depression-screening tools (HADS) ${ }^{21}$. Third, pSS and depression may share the common immune/inflammation dysfunction. Evidence indicated that immune/inflammation was a mediating pathway to both risk and neuroprogression in depression ${ }^{22,23}$. Current knowledge suggests that the interplay between genetic, environmental, hormonal, and neuropsychological factors underlies the pSS pathogenesis, leading to initiation and perpetuation of aberrant immune/inflammatory responses ${ }^{2}$. In a recent report, it was found that the surface expression of P2X7R, which has an important role in inflammation and immunity, on peripheral blood mononuclear cells was significantly higher in patients with anxiety/depression than in patients without anxiety/ depression ${ }^{24}$. In addition, a study in patients with pSS showed a higher proportion of patients with depression than patients without depression had serum antibody directed against N-methyl-D-aspartate receptor subtype NR2 levels above the cutoff value ${ }^{25}$. Fourth, previous studies have found an increased frequency of central nervous system white matter lesions (WML) in patients with $\mathrm{pSS}^{26}$. There is also growing evidence linking WML to depression ${ }^{27}$. The increased brain WML in patients with pSS may build up the subsequent depression.

In our study, patients with pSS were found to have 1.967 times greater risk of sleep disorder than the comparison cohort. Sleep disturbance has been found in the patients with pSS, with delayed sleep onset and excessive daytime sleepiness ${ }^{28,29}$. Moderate or severe sleep disturbances were reported by 49 out of 65 patients with pSS $(75 \%)^{30}$. However, the study found that the sleep disturbances were not associated with any clinical or laboratory variables in pSS subjects. Overnight polysomnography has demonstrated that patients with pSS had increased obstructive sleep apnea ${ }^{28}$ that may explain part of the sleep disturbance in such patients.

Increased depression, anxiety, and sleep disorder in pSS subjects could be a surveillance bias ${ }^{31}$ because patients with pSS are likely to exhibit a higher frequency of outpatient visits than the general population, leading to an earlier diagnosis of psychiatric disorders. To exclude this possibility, we conducted a subgroup analysis stratified according to the duration between the diagnosis of pSS and new-onset psychiatric disorders. The results indicated that incident depressive disorder, anxiety disorder, and sleep disorder were still

Personal non-commercial use only. The Journal of Rheumatology Copyright $@$ 2015. All rights reserved 
increased after the first year after a diagnosis of pSS. Therefore the increased risk of depressive disorder, anxiety disorder, and sleep disorder in patients with pSS in our current study was not attributable to surveillance bias.

In a nationwide population-based study of comorbidity profiles among patients with pSS in Taiwan, it was found that patients with pSS had higher psychotic disorder comorbidity than patients without $\mathrm{pSS}^{8}$. However, in our nationwide population-based study in Taiwan, no significantly increased risk of subsequent schizophrenia was observed after a diagnosis of pSS. The discrepancy between the 2 nationwide population-based studies in Taiwan may come from the difference in study design (retrospective cohort study vs cross-sectional study), and psychiatric diagnosis [ICD-9-CM codes for schizophrenia (295) vs the Elixhauser Comorbidity Index for psychosis (295-298, 299.1)].

The strength of our study is the use of a population-based dataset with enrollment of a large number of subjects that enabled us to trace the subsequent risk of psychiatric disorders in pSS subjects and controls. Further, the diagnoses of pSS and psychiatric disorders were done by specialists. A widespread skepticism prevails regarding the validity of diagnostic coding in medical claims, but previous epidemiological studies conducted with LHID have revealed results consistent with population-based surveys for several conditions $^{32}$. Finally, our study design included an unbiased participant selection process. Because participation in NHI is mandatory and all residents of Taiwan can access healthcare with low copayments, referral biases are low and followup compliance is high.

Certain limitations to our findings should, however, be considered. First, some patient information that may influence the risk of psychiatric disorders, such as medication, alcohol consumption, disease severity, and family history of psychiatric diseases, was not available for analysis. Thus, we were unable to control for these potentially confounding factors. Second, the severity and patterns of psychosocial stresses were unclear; these stresses might be related to other causes of psychiatric disorders after newly diagnosed pSS. Third, the diagnoses of pSS were identified using the ICD-9 codes from the database, and its prevalence may be underestimated because only subjects seeking medical evaluation could be identified, but this would most likely result in an underestimate of the association between pSS and psychiatric disorders. Finally, the data we obtained on pSS and psychiatric disorder diagnoses were highly reliable, and the diagnoses in NHI claims were primarily for administrative billing and did not undergo verification for scientific purposes. In studies using NHIRD, it was not clear how the diagnostic classification was performed. Therefore, the diagnostic accuracy could not be ascertained.

This nationwide population-based retrospective cohort study provides an initial glimpse into the development of psychiatric disorders after pSS diagnosis. Because affective conditions in pSS subjects are treatable conditions ${ }^{33,34}$ and have great effect on life quality, it is important to appropriately screen for and adequately treat these psychiatric disorders in this population, starting at disease onset.

\section{ACKNOWLEDGMENT}

We thank Emily Ting for English editing.

\section{REFERENCES}

1. Fox RI. Sjögren's syndrome. Lancet 2005;366:321-31

2. Mavragani CP, Moutsopoulos HM. Sjögren's syndrome. Annu Rev Pathol 2014;9:273-85.

3. Detweiler-Bedell JB, Friedman MA, Leventhal H, Miller IW, Leventhal EA. Integrating co-morbid depression and chronic physical disease management: identifying and resolving failures in self-regulation. Clin Psychol Rev 2008;28:1426-46.

4. Bergdahl J, Bergdahl M. Environmental illness: evaluation of salivary flow, symptoms, diseases, medications, and psychological factors. Acta Odontol Scand 2001;59:104-10.

5. Utset TO, Golden M, Siberry G, Kiri N, Crum RM, Petri M. Depressive symptoms in patients with systemic lupus erythematosus: association with central nervous system lupus and Sjogren's syndrome. J Rheumatol 1994;21:2039-45.

6. Stevenson HA, Jones ME, Rostron JL, Longman LP, Field EA. UK patients with primary Sjogren's syndrome are at increased risk from clinical depression. Gerodontology 2004;21:141-5.

7. Valtýsdóttir ST, Gudbjörnsson B, Lindqvist U, Hällgren R, Hetta J. Anxiety and depression in patients with primary Sjogren's syndrome. J Rheumatol 2000;27:165-9.

8. Kang JH, Lin HC. Comorbidities in patients with primary Sjogren's syndrome: a registry-based case-control study. J Rheumatol 2010;37:1188-94

9. Inal V, Kitapcioglu G, Karabulut G, Keser G, Kabasakal Y. Evaluation of quality of life in relation to anxiety and depression in primary Sjogren's syndrome. Mod Rheumatol 2010;20:588-97.

10. Cho HJ, Yoo JJ, Yun CY, Kang EH, Lee HJ, Hyon JY, et al. The EULAR Sjogren's syndrome patient reported index as an independent determinant of health-related quality of life in primary Sjogren's syndrome patients: in comparison with non-Sjogren's sicca patients. Rheumatology 2013;52:2208-17.

11. Lendrem D, Mitchell S, McMeekin P, Bowman S, Price E, Pease CT, et al. Health-related utility values of patients with primary Sjogren's syndrome and its predictors. Ann Rheum Dis 2014;73:1362-8.

12. Segal B, Thomas W, Rogers T, Leon JM, Hughes P, Patel D, et al. Prevalence, severity, and predictors of fatigue in subjects with primary Sjögren's syndrome. Arthritis Rheum 2008;59:1780-7.

13. Westhoff G, Dörner T, Zink A. Fatigue and depression predict physician visits and work disability in women with primary Sjögren's syndrome: results from a cohort study. Rheumatology 2012;51:262-9.

14. Hackett KL, Newton JL, Frith J, Elliott C, Lendrem D, Foggo H, et al. Impaired functional status in primary Sjogren's syndrome. Arthritis Care Res 2012;64:1760-4.

15. Wu CY, Chen YJ, Ho HJ, Hsu YC, Kuo KN, Wu MS, et al. Association between nucleoside analogues and risk of hepatitis B virus-related hepatocellular carcinoma recurrence following liver resection. JAMA 2012;308:1906-14.

16. Kang JH, Chen YH, Lin HC. Comorbidity profiles among patients with ankylosing spondylitis: a nationwide population-based study. Ann Rheum Dis 2010;69:1165-8.

17. Stojan G, Baer AN, Danoff SK. Pulmonary manifestations of Sjögren's syndrome. Curr Allergy Asthma Rep 2013;13:354-60.

Personal non-commercial use only. The Journal of Rheumatology Copyright $\odot$ 2015. All rights reserved. 
18. Ramos-Casals M, Brito-Zerón P, Sisó A, Vargas A, Ros E, Bove A, et al. High prevalence of serum metabolic alterations in primary Sjögren's syndrome: influence on clinical and immunological expression. J Rheumatol 2007;34:754-61.

19. Hyphantis T, Mantis D, Voulgari PV, Tsifetaki N, Drosos AA. The psychological defensive profile of primary Sjögren's syndrome patients and its relationship to health-related quality of life. Clin Exp Rheumatol 2011;29:485-93.

20. Karaiskos D, Mavragani CP, Sinno MH, Déchelotte P, Zintzaras E, Skopouli FN, et al. Psychopathological and personality features in primary Sjogren's syndrome - associations with autoantibodies to neuropeptides. Rheumatology 2010;49:1762-9.

21. Gandía M, Morales-Espinoza EM, Martín-González RM, Retamozo S, Kostov B, Belenguer-Prieto R, et al. Factors influencing dry mouth in patients with primary Sjögren syndrome: usefulness of the ESSPRI index. Oral Health Dent Manag 2014;13:402-7.

22. Berk M, Williams LJ, Jacka FN, O’Neil A, Pasco JA, Moylan S, et al. So depression is an inflammatory disease, but where does the inflammation come from? BMC Med 2013;11:200.

23. Dantzer R, O'Connor JC, Freund GG, Johnson RW, Kelley KW. From inflammation to sickness and depression: when the immune system subjugates the brain. Nat Rev Neurosci 2008;9:46-56.

24. Xie B, Chen Y, Zhang S, Wu X, Zhang Z, Peng Y, et al. The expression of $\mathrm{P} 2 \mathrm{X} 7$ receptors on peripheral blood mononuclear cells in patients with primary Sjögren's syndrome and its correlation with anxiety and depression. Clin Exp Rheumatol 2014;32:354-60.

25. Lauvsnes MB, Maroni SS, Appenzeller S, Beyer MK, Greve OJ, Kvaløy JT, et al. Memory dysfunction in primary Sjögren's syndrome is associated with anti-NR2 antibodies. Arthritis Rheum 2013;65:3209-17.
26. Akasbi M, Berenguer J, Saiz A, Brito-Zerón P, Pérez-De-Lis M, Bové A, et al. White matter abnormalities in primary Sjögren syndrome. QJM 2012;105:433-43.

27. Wang L, Leonards CO, Sterzer P, Ebinger M. White matter lesions and depression: a systematic review and meta-analysis. J Psychiatr Res 2014;56:56-64.

28. Usmani ZA, Hlavac M, Rischmueller M, Heraganahally SS, Hilditch CJ, Lester S, et al. Sleep disordered breathing in patients with primary Sjögren's syndrome: a group controlled study. Sleep Med 2012;13:1066-70.

29. Theander L, Strömbeck B, Mandl T, Theander E. Sleepiness or fatigue? Can we detect treatable causes of tiredness in primary Sjögren's syndrome? Rheumatology 2010;49:1177-83.

30. Tishler M, Barak Y, Paran D, Yaron M. Sleep disturbances, fibromyalgia and primary Sjögren's syndrome. Clin Exp Rheumatol 1997;15:71-4.

31. Haut ER, Pronovost PJ. Surveillance bias in outcomes reporting. JAMA 2011;305:2462-3.

32. Lin HC, Lin YJ, Liu TC, Chen CS, Chiu WT. Urbanization and stroke prevalence in Taiwan: analysis of a nationwide survey. J Urban Health 2007;84:604-14.

33. Ibn Yacoub Y, Rostom S, Laatiris A, Hajjaj-Hassouni N. Primary Sjögren's syndrome in Moroccan patients: characteristics, fatigue and quality of life. Rheumatol Int 2012;32:2637-43.

34. Wyszynski AA, Wyszynski B. Treatment of depression with fluoxetine in corticosteroid-dependent central nervous system Sjögren's syndrome. Psychosomatics 1993;34:173-7. 